\title{
Peso de Abate de Machos não-castrados para Produção do Bovino Jovem. 1. Desempenho em Confinamento e Custos de Produção
}

\author{
Geraldo Maria da Cruz ${ }^{1}$, Sérgio Novita Esteves ${ }^{2}$, Rymer Ramiz Tullio ${ }^{3}$, Maurício Mello de \\ Alencar ${ }^{4}$, Márcia Cristina de Sena Oliveira $^{5}$
}

\begin{abstract}
RESUMO - Com o objetivo de obter o peso adequado de abate de machos não-castrados e a economicidade da produção do bovino jovem, foi realizado na Embrapa Pecuária Sudeste um confinamento com 234 animais cruzados Blonde d’Aquitaine x Nelore (BN), Canchim x Nelore (CN), Limousin x Nelore (LN) e Piemontês x Nelore (PN) e puros Canchim (CA) e Nelore (NE) nos anos de 1994, 1995 e 1997. Ao início do experimento, os animais apresentavam 12 meses de idade; os BN, CA, CN e LN pesavam $265 \mathrm{~kg}$, os NE $214 \mathrm{~kg}$ e os PN 237 kg. Foram testados os pesos de abate (TRAT) de 400 (I), 440 (II) e $480 \mathrm{~kg}$ (III), exceto para os bovinos NE, que foram de 380, 410 e $440 \mathrm{~kg}$. A dieta, fornecida à vontade, possuía 13\% de proteína bruta e 70\% de nutrientes digestíveis totais, à base de 50\% de silagem de milho e $50 \%$ concentrado, na base seca. As pesagens dos animais foram realizadas após jejum de 16 horas. Os dados foram submetidos à análise de variância e as médias, comparadas pelo SNK. As médias estimadas para ganho diário de peso (GDP), consumo diário de matéria seca (CMS), em kg e em percentagem do peso vivo, eficiência de conversão alimentar (ECA) e período de confinamento foram 1,56; 1,49 e 1,44 \pm 0,03 kg; 9,01; 9,01 e 9,21 \pm 0,13 kg; 2,58; 2,44 e 2,45 \pm 0,04\%; 5,92; 6,26 e 6,49 \pm 0,12; e 71,3; 95,6 e 115,3 \pm 2,1 dias, respectivamente, para os tratamentos I, II e III. Os efeitos de ano, de grupo genético e de TRAT foram significativos para GDP, CMS e ECA. As interações entre grupo genético e TRAT não foram significativas. Houve aumento no custo da arroba produzida, de R \$ 39,14 para R\$ 40,31 ou R \$4,49, com o aumento do peso de abate de 400 para 440 ou $480 \mathrm{~kg}$ de peso vivo, respectivamente. A rentabilidade mensal reduziu de $1,0 \%$ para $0,7 \%$ ou $0,6 \%$ para os TRAT I, II ou III, respectivamente.
\end{abstract}

Palavras-chave: consumo de alimentos, conversão alimentar, cruzados, ganho de peso

\section{Slaughter Weights for the Production of Young Bull Cattle. 1. Performance on Feedlot and Cost of Production}

\begin{abstract}
To obtain the adequate slaughter weights and costs of production of young bulls, a feedlot study was conducted at Embrapa Pecuaria Sudeste, with 234 crossbred Blonde d'Aquitaine x Nellore (BN); Canchim x Nellore (CN); Limousin x Nellore (LN); Piedmontese x Nellore (PN); and straightbred Canchim (CA) and Nellore (NE) in 1994, 1995 and 1997. At the beggining of the experiment, the animals had 12 months of age and the BN, CA, CN, LN weighed $265 \mathrm{~kg}$, the NE $214 \mathrm{~kg}$ and the PN $237 \mathrm{~kg}$. The slaughter weights (TRAT) of 400 (I); 440 (II) and $480 \mathrm{~kg}$ (III), except for NE bulls (380, 410 and $440 \mathrm{~kg}$ ), were tested. A diet with 13\% crude protein and $70 \%$ total digestible nutrients, based on $50 \%$ whole plant corn silage and 50\% concentrate, on a dry matter basis, was fed ad libitum. The measurement of weights of the animals were performed after fasting for 16 hours. Data were submitted to the analysis of variance and the means compared by SNK tests. The estimated means of daily weight gain (DWG), daily dry matter intake (DMI), kg and as a percentage of live weight, the feed:gain ratio (FG) and days on feed for TRAT I; II and III were 1.56; 1.49 and $1.44 \pm 0.03 \mathrm{~kg}$; 9.01 ; 9.01 and $9.21 \pm 0.13 \mathrm{~kg}$; 2.58; 2.44 and $2.45 \pm 0.04 \%$; 5.92; 6.26 and $6.49 \pm 0.12$; 71.3; 95.6 and $115.3 \pm 2.1$ days, respectively. The effects of year, genetic group and TRAT were significant for DWG, DMI and FG. The interactions between genetic group and TRAT were not significant. There was an increase in the cost of production of $15 \mathrm{~kg}$ of carcass in the feedlot from 39.14 to 40.31 or 40.49 reais with the increase in the slaughter weight from 400 to 440 or $480 \mathrm{~kg}$ live weigth, respectively. The monthly net income of the capital invested was reduced from $1.0 \%$ to $0.7 \%$ or $0.6 \%$ for TRAT I, II or III, respectively.
\end{abstract}

Key words: crossbred, feed intake, feed: gain ratio, weight gain

\footnotetext{
${ }^{1}$ Pesquisador da Embrapa Pecuária Sudeste, C.P. 339, CEP: 13560-970, São Carlos, SP. E.mail: geraldo@cppse.embrapa.br 2 Pesquisador da Embrapa Pecuária Sudeste, C.P. 339, CEP: 13560-970, São Carlos, SP. E.mail: sergio@cppse.embrapa.br

${ }^{3}$ Pesquisador da Embrapa Pecuária Sudeste, pós-graduando em Zootecnia FCAV/UNESP, Jaboticabal, SP.

E.mail: rymer@cppse.embrapa.br

${ }^{4}$ Pesquisador da Embrapa Pecuária Sudeste, C.P. 339, CEP: 13560-970, São Carlos, SP. Bolsista CNPq. E.mail: mauricio@cppse.embrapa.br

${ }^{5}$ Pesquisador da Embrapa Pecuária Sudeste, C.P. 339, CEP: 13560-970, São Carlos, SP. E.mail: marcia@cppse.embrapa.br
} 


\section{Introdução}

Os padrões de produção intensiva de carne bovina, na década de 70, nos países desenvolvidos, já recomendavam o abate de machos com menos de 18 meses de idade (Preston \& Willis, 1974). Estudos de pesos de abate, visando melhor desempenho em confinamento e características desejáveis de carcaça foram efetuados, entre outros, com machos nãocastrados da raça Holandesa (Bailey et al., 1985), cruzados Blonde d'Aquitaine x Charolês (Patterson et al., 1994), Nelore e cruzados Limousin x Nelore e Marchigiana x Nelore (Galvão et al., 1991), Nelore e cruzados Holandês x Nelore e bimestiços FleckviehAngus x Nelore (Jorge et al., 1997), e machos castrados Angus e cruzados Angus x Brahman (Huffman et al., 1990).

As dificuldades para abater animais jovens ainda persistem nas condições brasileiras (Galvão et al., 1991; Euclides Filho et al., 1997; Jorge et al., 1997), uma vez que os estudos de peso de abate citados demonstraram que o manejo dos animais visava o abate entre 24 e 30 meses de idade. Além da idade elevada para padrões internacionais, mostraram também que os animais cruzados não atingiram a terminação adequada de, no mínimo, 3 mm de espessura de gordura externa (Mattos, 1995).

Nos Estados Unidos, Urick et al. (1991) utilizando dados de cruzamentos e confinamento de bovinos da década de 70, demonstraram diferenças entre grupos genéticos de animais cruzados para ganho de peso, consumo de alimentos e conversão energética (Mcal consumida/kg de ganho de peso), considerando três diferentes pontos de abate (382 dias de idade, $400 \mathrm{~kg}$ de peso vivo ou 12,7 $\mathrm{mm}$ de espessura de gordura externa). Galvão et al. (1991) obtiveram redução de 9,6\% na eficiência de conversão alimentar, com o aumento de peso de abate de 450 para $550 \mathrm{~kg}$ em bovinos não-castrados, sendo que outros autores não obtiveram redução na eficiência, trabalhando com animais não-castrados da raça Holandesa (Levy et al., 1975) e bovinos e bubalinos entre 400 e $500 \mathrm{~kg}$ de peso vivo (Jorge et al., 1997).

Hansen \& Zinn (1968), citados por Preston \& Willis (1974), demonstraram que animais abatidos entre 272 e $454 \mathrm{~kg}$ de peso vivo não diferiram quanto à porcentagem de carne de primeira ou porcentagem total de carne comestível na carcaça. Contudo, no trabalho de Breidenstein et al. (1965), também citado por Preston \& Willis (1974), com incrementos no peso de abate de 307 para 386; 466 ou 545 kg, ocorreram aumentos no rendimento de carcaça quente de 54,5 para 56,$1 ; 57,4$ ou $59,1 \%$ e redução na percentagem de carne de primeira e no rendimento de carne comestível.

Os objetivos deste estudo foram comparar o desempenho em confinamento de machos não-castrados abatidos aos 400, 440 ou $480 \mathrm{~kg}$ de peso vivo, e verificar a economicidade da produção do bovino jovem para abate dos 15 aos 18 meses de idade.

\section{Material e Métodos}

O trabalho foi desenvolvido na Embrapa Pecuária Sudeste com animais cruzados $\left(3 /{ }_{8}\right)$ 1/2 Blonde d'Aquitaine x (5/8) $1 / 2$ Nelore (BN), $1 / 2$ Canchim x $1 / 2$ Nelore (CN), $1 / 2$ Limousin x $1 / 2$ Nelore (LN) e $1 / 2$ Piemontês $x$ 1 $1 / 2$ Nelore (PN) e puros Canchim (CA) e Nelore (NE) nos anos de 1994, 1995 e 1997, sendo que cada grupo genético participou em dois anos, exceto $\mathrm{BN}$, que foram confinados nos três anos. Animais $3 /{ }_{8}$ Blonde d'Aquitaine $\mathrm{x} 5 / 8$ Nelore foram utilizados em 1994, enquanto animais $1 / 2$ Blonde d'Aquitaine x 1/2 Nelore foram utilizados em 1995 e 1997. Os animais CA e CN pertenciam ao rebanho da Embrapa Pecuária Sudeste e os animais dos outros grupos genéticos, a rebanhos de produtores particulares, de propriedades localizadas nos municípios de Marabá, PA e Avaré, Bariri e Santa Fé do Sul, SP.

Um total de 234 animais foi utilizado para o estudo de desempenho em confinamento, sendo que um animal BN foi sacrificado após acidente de manejo no final do período de adaptação. Ao início do período pré-experimental, os animais apresentavam 12 meses de idade e os BN, CA, CN e LN pesavam, em média, $265 \mathrm{~kg}$, os NE $214 \mathrm{~kg}$ e os PN $237 \mathrm{~kg}$. Lotes de seis animais de cada grupo genético (GG) foram alocados nos tratamentos (TRAT) que são os pesos de abate de 400 (I), 440 (II) e $480 \mathrm{~kg}$ (III), exceto para os bovinos NE que foram de 380 , 410 e $440 \mathrm{~kg}$. Os animais receberam, ad libitum, uma dieta com $13 \%$ de proteína bruta e $70 \%$ de nutrientes digestíveis totais, à base de $50 \%$ de silagem de milho, 29,2\% de milho em grão moído, $9,1 \%$ de farelo de soja, $9,9 \%$ de farelo de trigo, $0,8 \%$ de calcário calcítico e $1 \%$ de sal mineral, na base seca. As quantidades ofertadas diariamente de silagem de milho e de concentrado foram reajustadas de acordo com o consumo, pesadas e misturadas manualmente dentro dos cochos, às 8 e 16 h. As

\section{R. Bras. Zootec., v.33, n.3, p.635-645, 2004}


sobras de alimentos foram pesadas uma vez por dia, sempre no período da manhã.

O manejo sanitário dos animais incluiu exames de tuberculose (tuberculinização com PPD bovino), brucelose (soroaglutinação) e vacinações contra febre aftosa. O tratamento contra helmintos grastrintestinais foi feito com base nos resultados dos exames de fezes e o controle de carrapatos, com base na avaliação empírica da infestação.

Os teores de matéria seca dos alimentos e das sobras foram determinados a cada 15 dias, em estufa com circulação forçada de ar a $60^{\circ} \mathrm{C}$, por 72 horas (Silva, 1990). As médias das análises químicobromatológicas das amostras de silagem de milho e do concentrado, realizadas de acordo com Goering \& Van Soest (1970) e Silva (1990), estão apresentadas na Tabela 1.

O peso vivo dos animais foi obtido a intervalos de
28 dias, após um período preliminar de 31, 27 e 36 dias, nos anos de 94, 95 e 97, respectivamente, após jejum de água e alimentos de 16 horas. Animais foram escolhidos individualmente para abate, assim que atingiram o peso-meta do TRAT. Os ganhos de peso foram calculados para o período experimental, para cada animal, e o consumo diário de matéria seca (CMS) e a eficiência de conversão alimentar (ECA), em termos de quilograma de matéria seca ingerida por quilograma de ganho de peso, para cada baia de seis animais.

Os dados de ganho de peso foram submetidos à análise de variância pelo procedimento GLM do SAS (1999), considerando os efeitos das variáveis ano, GG, TRAT e a interação GG X TRAT, e também aplicando o modelo com as variáveis ano e TRAT para cada GG separadamente. Para análise dos dados de CMS e ECA, cada lote com seis animais foi

Tabela 1 - Média da composição químico-bromatológica da silagem de milho e do concentrado da dieta oferecida aos bovinos em confinamento, em percentagem da matéria seca

Table 1 - Average chemical composition of whole plant corn silage and concentrate offered in diets of cattle in feedlot, in dry matter basis

\begin{tabular}{|c|c|c|}
\hline $\begin{array}{l}\text { Análise } \\
\text { Analysis }\end{array}$ & $\begin{array}{l}\text { Silagem de milho } \\
\text { Whole plant corn silage }\end{array}$ & $\begin{array}{c}\text { Concentrado } \\
\text { Concentrate }\end{array}$ \\
\hline Matéria seca & 36,87 & 88,89 \\
\hline Dry matter & & \\
\hline Proteína bruta & 7,15 & 19,38 \\
\hline Crude protein & & \\
\hline $\begin{array}{l}\text { Fibra em detergente neutro (FDN) } \\
\text { Neutral detergent fiber }\end{array}$ & 50,00 & 28,87 \\
\hline $\begin{array}{l}\text { Fibra em detergente ácido (FDA) } \\
\text { Acid detergent fiber }\end{array}$ & 28,17 & 8,81 \\
\hline $\begin{array}{l}\text { Nitrogênio insolúvel na FDA }{ }^{1} \\
\text { Acid detergent nitrogen }\end{array}$ & 8,19 & 3,95 \\
\hline $\begin{array}{l}\text { Celulose } \\
\text { Cellulose }\end{array}$ & 24,92 & 7,54 \\
\hline $\begin{array}{l}\text { Lignina } \\
\text { Lignin }\end{array}$ & 3,89 & 1,87 \\
\hline $\begin{array}{l}\text { Digestibilidade in vitro da matéria seca } \\
\text { In vitro dry matter digestibility }\end{array}$ & 65,43 & 88,64 \\
\hline $\mathrm{pH}$ & 3,88 & - \\
\hline $\begin{array}{l}\text { Matéria mineral } \\
\text { Ash }\end{array}$ & 3,09 & 6,18 \\
\hline $\begin{array}{l}\text { Cálcio } \\
\text { Calcium }\end{array}$ & 0,14 & 0,76 \\
\hline $\begin{array}{l}\text { Fósforo } \\
\text { Phosphorus }\end{array}$ & 0,16 & 0,72 \\
\hline $\begin{array}{l}\text { Amônia }{ }^{1} \\
\text { Ammonium }^{1}\end{array}$ & 5,71 & - \\
\hline
\end{tabular}

${ }^{1}$ Expresso em percentagem do nitrogênio total (Expressed as percentage of total nitrogen). 
utilizado como parcela experimental. As médias estimadas foram comparadas pelo teste Student Newman-Keuls (SNK).

Para a análise econômica do confinamento, foi realizado um levantamento de preços de compra e venda de animais, de mão-de-obra, de hora-máquina e dos insumos (ingredientes da ração, vacinas e medicamentos), do Instituto de Economia Agrícola (IEA) (2002) relativos ao ano de 2001. O custo de produção da silagem de milho foi obtido do Boletim do Leite (2001). Os preços dos ingredientes da ração foram R\$ 43,45; 172,00; 490,00; 225,00; 55,00 e 400,00 por tonelada de silagem de milho, de milho em grão, de farelo de soja, de farelo de trigo, de calcário calcítico e de sal mineralizado, respectivamente. Os custos de compra de bezerros ( $\mathrm{R} \$ 1,56 / \mathrm{kg}$ de peso vivo) aplicado ao peso vivo dos animais na entrada no confinamento (junho) e a média dos preços de venda do boi gordo dos meses de setembro, outubro e novembro (R\$43,00/@ de carcaça quente) foram utilizados nos cálculos.

Foi considerado que uma pessoa é capaz de preparar ração e fornecer alimentos para um lote de 78 animais ao custo diário de R $\$ 12,00$ (IEA, 2002) e custo de um salário-mínimo para assistência veterinária por lote de 78 animais. O custo das instalações específicas (cocho para fornecimento de alimentos, bebedouro e cercas) foi depreciado por dez anos com taxa de juros de $6 \%$ ao ano e custo de manutenção de $4 \%$ ao ano. A análise econômica considerou ainda juros de $6 \%$ ao ano sobre o capital utilizado para custeio da atividade, não incluindo o capital investido em animais. No cálculo da rentabilidade do confinamento, foi considerado desconto do custo de comercialização, ou seja, taxas do Funrural (previdência rural) (2,3\%) e Guia de Recolhimento Estadual (R\$1,26/animal).

\section{Resultados e Discussão}

A média estimada do desempenho dos animais em confinamento pode ser observada na Tabela 2.

A média de peso vivo no início da fase experimental foi 295,7 $\pm 2,8 \mathrm{~kg}$, não havendo diferenças entre tratamentos; porém ocorreram $(\mathrm{P}<0,05)$ diferenças quanto ao ano e GG. As médias estimadas de peso vivo, no início da fase experimental, foram de 336, 284 e $270 \pm 3,2 \mathrm{~kg}$ para os anos 94, 95 e 97, respectivamente e 324; 314; 313; 310; 266; e $236 \pm$ 4,3 kg para os grupos genéticos LN, CN, BN, CA, PN e NE, respectivamente. As médias estimadas de ganho diário de peso vivo foram de 1,56; 1,49 e 1,44 \pm $0,03 \mathrm{~kg}$ para os TRAT I, II e III, respectivamente. Pode-se observar que houve redução gradativa de

Tabela 2 - Média estimada do desempenho de machos não-castrados em confinamento, de acordo com o peso de abate ${ }^{1}$

Table 2 - Estimated means of performance of young bulls in feedlot, according to the slaughter weight

\begin{tabular}{|c|c|c|c|c|}
\hline & \multicolumn{3}{|c|}{$\begin{array}{l}\text { Peso de abate, } \mathrm{kg} \\
\text { Slaughter weight, } \mathrm{kg} \\
\end{array}$} & \multirow[t]{2}{*}{$\begin{array}{l}\text { Erro-padrão } \\
\text { Standard error }\end{array}$} \\
\hline & $400(\mathrm{I})$ & 440 (II) & 480 (III) & \\
\hline Peso vivo, kg & $295,9^{a}$ & $294,2^{\mathrm{a}}$ & $294,6^{\mathrm{a}}$ & 2,8 \\
\hline \multicolumn{5}{|l|}{ Live weight, $\mathrm{kg}$} \\
\hline Dias em confinamento & $71,3^{\mathrm{C}}$ & $95,6^{\mathrm{b}}$ & $115,3^{\mathrm{a}}$ & 2,1 \\
\hline Ganho diário de peso (GDP), kg & \multicolumn{4}{|c|}{ Average daily gain (ADG), $\mathrm{kg}$} \\
\hline $\begin{array}{l}\text { Consumo diário de matéria seca (CMS })^{2}, \mathrm{~kg} \\
\text { Average daily dry matter intake }(\mathrm{DMI})^{2}, \mathrm{~kg}\end{array}$ & $9,01^{\mathrm{a}}$ & $9,01^{\mathrm{a}}$ & $9,21^{\mathrm{a}}$ & 0,13 \\
\hline $\begin{array}{l}\text { Consumo diário de matéria seca }{ }^{2}, \% \text { peso vivo } \\
\text { Average daily dry matter intake }^{2}, \% \text { of live weight }\end{array}$ & $2,58^{\mathrm{a}}$ & $2,49^{\mathrm{ab}}$ & $2,44^{b}$ & 0,04 \\
\hline $\begin{array}{l}\text { Eficiência de conversão alimentar², CMS/GDP } \\
\text { Feed:gain ratio }{ }^{2}, D M I / A D G\end{array}$ & $5,92^{\mathrm{b}}$ & $6,26^{\mathrm{ab}}$ & $6,49^{\mathrm{a}}$ & 0,12 \\
\hline \multicolumn{5}{|c|}{$1 \quad$ Pesos previstos para abate dos animais da raça Nelore foram 380, 410 e $440 \mathrm{~kg}$. } \\
\hline \multicolumn{5}{|c|}{1 Designed slaughter weights of Nellore cattle were 380,410 and $440 \mathrm{~kg}$. } \\
\hline \multicolumn{5}{|c|}{2 Média de baias com seis animais cada uma. } \\
\hline $\begin{array}{l}2 \quad \text { Average of pens with six animals each one. } \\
\text { abc Médias seguidas de letras iguais na mesma linh } \\
\text { abc Means in the same row followed by the same letter. }\end{array}$ & difor & $>0,05) p$ & ste SNK. & \\
\hline
\end{tabular}

R. Bras. Zootec., v.33, n.3, p.635-645, 2004 
ganho de peso, à medida que se elevou o peso de abate, sendo a diferença estatisticamente significativa $(\mathrm{P}<0,05)$ entre os TRAT I e III, isto é, animais abatidos aos 400 e $480 \mathrm{~kg}$ de peso vivo. Bailey et al. (1985), trabalhando com machos não-castrados da raça Holandesa abatidos aos 340, 470 e $600 \mathrm{~kg}$ de peso vivo, observaram redução do ganho de peso vivo de 1,30 para 1,26 e 1,18 kg/dia, quando os pesos de abate aumentaram de 340 para 470 ou 600 kg, respectivamente. Contudo, Galvão et al. (1991) não encontraram diferenças nos ganhos diários de peso vivo com alteração no peso de abate de 450 para 500 ou $550 \mathrm{~kg}$ dos animais Nelore x Marchigiana (1,11 kg) ou Nelore x Limousin (1,20 kg) e de 405 para 450 ou $500 \mathrm{~kg}$ dos animais Nelore $(0,95 \mathrm{~kg})$, trabalhando com animais não-castrados, assim como Broadbent (1976), que verificou $0,8 \mathrm{~kg} / \mathrm{dia}$, trabalhando com animais castrados de raças britânicas abatidos aos 418; 458 e $496 \mathrm{~kg}$. Huffman et al. (1990) obtiveram elevados ganhos diários de peso vivo (1,69 kg) e semelhantes entre quatro pontos de acabamento das carcaças, variando de 440 a $507 \mathrm{~kg}$ de peso vivo, em animais Angus e cruzados Angus x Brahman.

Segundo Jorge et al. (1997), o maior ganho diário de peso em animais abatidos com $550 \mathrm{~kg}$ em relação aos animais abatidos aos 500 kg (Holandês x Nelore e bimestiço Nelore x Angus x Fleckvieh) ou 450 para 500 kg (Nelore e búfalos) não era esperado. Levy et al. (1975) também observaram aumento do ganho de peso vivo, com bovinos da raça Holandesa não-castrados, com os incrementos nos pesos de abate de 400 para 450 e $500 \mathrm{~kg}$. Patterson et al. (1994) verificaram tendência para redução do ganho de peso vivo com o aumento do peso de abate de 550 para 625 e $700 \mathrm{~kg}$ para animais não-castrados (7/8 de raças continentais) e afirmaram que o peso vivo de $550 \mathrm{~kg}$ já estava acima do ponto de inflexão da curva de ganho de peso, embora tenham utilizado grupos genéticos de maturação tardia.

A análise conjunta das 233 observações (três anos e seis grupos genéticos) revelou ainda que houve diferenças $(P<0,05)$ entre $G G$ e não houve efeito de ano e interação $(P>0,05)$ entre GG e TRAT para a variável ganho de peso. As médias estimadas dos ganhos diários de peso para $\mathrm{LN}, \mathrm{CN}, \mathrm{BN}, \mathrm{CA}$, PN e NE, foram 1,$70 ; 1,47 ; 1,55 ; 1,66 ; 1,48$ e $1,12 \pm$ $0,04 \mathrm{~kg}$, respectivamente. Apesar de a interação não ter sido significativa, foi realizada análise estatística para cada GG separadamente, devido à grande diferença observada entre GG e a desuniformidade de distribuição dos animais dos diferentes GG nos diferentes anos (94, 95 e 97). Os resultados desta análise estão apresentados na Tabela 3. Existiu tendência de redução de ganho de peso, com o aumento dos pesos de abate, nos limites

Tabela 3 - Média estimada de ganho diário de peso de machos não-castrados em confinamento, por grupo genético, de acordo com o peso de abate, em kg

Table 3 - Estimated means of daily live weight gain of young bull cattle in feedlot, according to the genetic group within slaughter weight, $\mathrm{kg}$

\begin{tabular}{|c|c|c|c|c|}
\hline & \multicolumn{3}{|c|}{$\begin{array}{l}\text { Peso de abate, kg } \\
\text { Slaughter weight, } k g\end{array}$} & \multirow[t]{2}{*}{$\begin{array}{l}\text { Erro-padrão } \\
\text { Standard error }\end{array}$} \\
\hline & 400 (I) & 440 (II) & 480 (III) & \\
\hline $\begin{array}{l}\text { Blonde d'Aquitaine x Nelore } \\
\text { Blonde d'Aquitaine x Nellore }\end{array}$ & $1,57^{\mathrm{a}}$ & $1,53^{\mathrm{a}}$ & $1,54^{\mathrm{a}}$ & 0,06 \\
\hline $\begin{array}{l}\text { Canchim } \\
\text { Canchim }\end{array}$ & $1,83^{\mathrm{a}}$ & $1,60^{\mathrm{a}}$ & $1,55^{\mathrm{a}}$ & 0,10 \\
\hline $\begin{array}{l}\text { Canchim x Nelore } \\
\text { Canchim } x \text { Nellore }\end{array}$ & $1,64^{\mathrm{a}}$ & $1,38^{\mathrm{a}}$ & $1,40^{\mathrm{a}}$ & 0,08 \\
\hline $\begin{array}{l}\text { Limousin } x \text { Nelore } \\
\text { Limousin } \times \text { Nellore }\end{array}$ & $1,70^{\mathrm{ab}}$ & $1,80^{\mathrm{a}}$ & $1,58^{b}$ & 0,06 \\
\hline $\begin{array}{l}\text { Piemontês x Nelore } \\
\text { Piedmontese x Nellore }\end{array}$ & $1,47^{\mathrm{a}}$ & $1,48^{\mathrm{a}}$ & $1,49^{a}$ & 0,05 \\
\hline $\begin{array}{l}\text { Nelore }^{1} \\
\text { Nellore }^{1}\end{array}$ & $1,13^{\mathrm{a}}$ & $1,12^{\mathrm{a}}$ & $1,11^{\mathrm{a}}$ & 0,06 \\
\hline
\end{tabular}

R. Bras. Zootec., v.33, n.3, p.635-645, 2004 
de 400 a $480 \mathrm{~kg}$ de peso vivo, nos GGCA, CN eLN, sendo significativo $(\mathrm{P}<0,05)$ apenas para os cruzados $\mathrm{LN}$.

O tempo necessário para os animais atingirem os pesos predeterminados para abate aumentou $(\mathrm{P}<0,05)$ a cada incremento de peso de abate de 400 para $480 \mathrm{~kg}$. As médias estimadas do período de confinamento foram 71,3; 95,6 e 115,3 \pm 2,1 dias, para os TRAT I, II e III, respectivamente, além do período pré-experimental, que variou de 27 a 36 dias, dependendo do ano. Dessa forma, os valores acima representam a média dos tempos individuais de confinamento de cada animal durante o período experimental. Também ocorreram efeitos de ano e GG com relação ao período de confinamento. As médias estimadas foram 63,3; 103,0 e 112,6 \pm 2,3 dias para os anos 94; 95 e 97, respectivamente, e 70,9; 82,9; 81,8; 80,9; 114,6 e 136,8 \pm 3,2 dias para os GG LN, CN, BN, CA, PN e NE, respectivamente. Os tempos de confinamento diferentes $(\mathrm{P}<0,05)$ entre os três anos se justifica em virtude dos maiores pesos vivos no início dos confinamentos de $94 \mathrm{em}$ relação aos anos de 95 e de 95 em relação a 97, já que não houve efeito $(\mathrm{P}>0,05)$ do ano de confinamento sobre o ganho diário de peso.

O CMS de alimentos não foi alterado $(\mathrm{P}>0,05)$ com os incrementos nos pesos de abate dos animais entre 400 e $480 \mathrm{~kg}$ de peso vivo, ocorrendo efeitos significativos $(\mathrm{P}<0,05)$ de ano e GG sobre essa variável. As médias estimadas dos CMS para os TRAT I, II e III foram 9,01; 9,01 e 9,21 \pm 0,13 kg/ animal/dia; para os anos 94, 95 e 97 foram 10,18; 8,86 e 8,23 kg/animal/dia; e para os animais LN, CN, BN, CA, PN e NE foram de 10,23; 9,25; 9,13; 9,48; 8,78 e 7,56 \pm 0,18 kg/animal/dia, respectivamente. Estes consumos foram maiores $(\mathrm{P}<0,05)$ para os animais $\mathrm{LN}$ e menores $(\mathrm{P}<0,05)$ para os animais $\mathrm{NE}$, sendo que os demais GG apresentaram consumos intermediários e semelhantes ( $\mathrm{P}>0,05)$. Levy et al. (1975), Bailey et al. (1985), Galvão et al. (1991) e Jorge et al. (1997) observaram incrementos nos CMS das dietas, com o aumento do peso de abate, enquanto Patterson et al. (1994) observaram apenas tendência de aumento do consumo de energia metabolizável. Quanto às diferenças relativas à variável ano, entre outros efeitos, deve-se ressaltar que ocorreram diferenças quanto ao peso inicial e idade dos animais. Cruz (2000), revisando a literatura, verificou que ocorreram acréscimos de 8,6 e 15,7\% nos CMS das dietas de bovinos em confinamento, quando a idade inicial passou de 10 para 16 ou de 16 para 20 meses.
Quando o consumo de alimentos foi expresso em percentagem do peso vivo (CMSPV), os valores obtidos foram 2,58; 2,49 e 2,44 $\pm 0,04 \%$, para os TRAT I, II e III, respectivamente. A redução do CMSPV foi significativa $(\mathrm{P}<0,05)$ entre os TRAT I e III. Pode-se observar que os animais não tiveram a capacidade de aumentar o consumo de matéria seca de alimentos proporcionalmente aos incrementos nos pesos de abate, sendo, provavelmente, a principal causa da redução dos ganhos diários de peso verificados no presente trabalho. Bailey et al. (1985) encontraram CMSPV de 2,28; 2,15 e 1,98\% para os pesos de abate de 340; 470 e $600 \mathrm{~kg}$, respectivamente. Galvão et al. (1991) e Jorge et al. (1997), por sua vez, não observaram diferenças nos consumos de matéria seca, expressos em percentagem do peso corporal vazio. A análise estatística de CMSPV mostrou efeito significativo $(\mathrm{P}<0,05)$ para ano, mas não houve efeito $(\mathrm{P}>0,05)$ para $G \mathrm{G}$ e para a interação GG x TRAT.

As médias estimadas dos CMSPV para os anos de 94, 95 e 97 foram 2,65; 2,49 e 2,37\%, respectivamente. Então, mesmo corrigindo os CMS para as médias de peso vivo, os efeitos permaneceram, demonstrando que a diferença de idade inicial entre anos pode ter sido um fator importante. As médias de idade inicial foram 420, 366 e 316 dias para os anos 94, 95 e 97, respectivamente. A qualidade da dieta, mostrada na Tabela 1, permaneceu uniforme durante todo o trabalho. As silagens de milho possuíam entre 35 e $40 \%$ de grãos na matéria seca ensilada, apresentando fermentação e análises químico-bromatológicas dentro dos padrões de qualidade para esta forragem (McCullough, 1978; Vilela, 1998).

A análise da ECA, expressa em quilograma de matéria seca consumida por quilograma de ganho de peso, mostrou efeitos significativos $(\mathrm{P}<0,05)$ para ano, TRAT e GG, porém não houve efeito $(\mathrm{P}>0,05)$ para a interação GG x TRAT. As médias estimadas da ECA, para os TRAT I, II e III, foram de 5,92; 6,26 e 6,49 $\pm 0,12$; para os anos de 94,95 e 97, de 6,48; 6,21 e 5,99; e para os animais LN, CN, BN, CA; PN e NE, de 6,09; 6,57; 6,02; 5,94; 6,03 e 6,81, respectivamente. Pode ser observada redução gradativa de ECA, à medida que se aumentou o peso de abate, sendo significativa $(\mathrm{P}<0,05)$ entre os TRAT I e III. Os incrementos nos pesos de abate de 400 para $440 \mathrm{~kg}$ e de 440 para $480 \mathrm{~kg}$, geralmente, requerem aumentos na quantidade de energia para mantença (NRC, 1996); contudo, estes não foram

\section{R. Bras. Zootec., v.33, n.3, p.635-645, 2004}


acompanhados, como citado anteriormente, por incrementos proporcionais no CMS de alimentos, causando redução no ganho diário de peso.

Diferenças na composição do ganho de peso (NRC, 1996), com incrementos nos pesos de abate e na duração dos períodos de confinamento, também podem ter contribuído para redução da ECA, uma vez que a gordura possui um valor calórico 2,25 vezes maior que a proteína e a deposição de gordura nos tecidos geralmente é acompanhada de redução no teor de água, diminuindo ainda mais o ganho de peso. Semelhantemente ao observado no presente estudo, Bailey et al. (1985) encontraram ECA de 4,72; 5,68 e 6,73 para bovinos da raça Holandesa abatidos aos 340; 470 e $600 \mathrm{~kg}$, respectivamente. Patterson et al. (1994) afirmaram que a redução do ganho de peso por unidade de energia metabolizável consumida, com o aumento do peso de abate, foi causado, principalmente, pelo aumento da energia de mantença em relação ao consumo de energia metabolizável e aumentos na gordura da carcaça e gordura interna; enquanto Galvão et al. (1991) mostraram redução na ECA de 9,6\%, porém, a diferença não foi estatisticamente significativa. Jorge et al. (1997) não encontraram diferença na conversão alimentar, expressa em relação ao peso corporal vazio.

O efeito de ano sobre a ECA pode ser atribuído ao binômio peso e idade inicial, que foram diferentes entre os anos avaliados. Cruz (2000), revisando a literatura, verificou que bovinos que entraram nos experimentos com mais de 20 meses de idade tiveram conversão alimentar $40 \%$ mais elevada que aquela observada para animais mais jovens (7 a 17 meses).

O efeito de GG sobre a ECA mostra que animais NE foram menos eficientes em relação ao grupo CA e aos cruzados, exceto CN. Animais NE foram abatidos com maior espessura de gordura externa que os demais grupos genéticos, sendo importante fator para a redução na eficiência da conversão alimentar.

O resultado da análise econômica por TRAT apresentado na Tabela 4 demonstrou que houve aumento no custo da arroba produzida no confinamento, de $\mathrm{R} \$ 39,14$ para $\mathrm{R} \$ 40,31$ ou $\mathrm{R} \$ 40,49$, com o aumento do peso de abate de 400 para 440 ou $480 \mathrm{~kg}$ de peso vivo, respectivamente. A rentabilidade mensal foi reduzida de 1,0 para $0,7 \%$ ou para $0,6 \%$ no abate dos animais aos 400,440 ou $480 \mathrm{~kg}$, respectivamente. O incremento dos custos variáveis, com o aumento do peso de abate, ocorreu principalmente em função de acréscimos nos custos com alimentos, uma vez que a eficiência de conversão alimentar foi reduzida, à medida que se aumentou o período de confinamento. Também ocorreram incrementos dos juros sobre custeio da atividade de 1,7 para 2,1 ou 2,4\%, com o aumento do peso de abate de 400 para 440 ou $480 \mathrm{~kg}$ de peso vivo, respectivamente.

Os custos com alimentos, mão-de-obra e máquinas para alimentação dos animais representaram 95,1\% dos custos do confinamento de animais jovens, citados na Tabela 4. Os custos totais (custos variáveis + custo dos animais + custos das instalações) da arroba de carcaça vendida, sem considerar os custos de comercialização, foram R\$ 40,51; R \$ 40,81 e R\$ 40,74, para os TRAT I, II e III, respectivamente. O capital investido na compra de animais representou 64,$01 ; 58,78$ e $54,62 \%$ e o custo dos alimentos fornecidos aos animais, 29,83; 34,29 e 35,97\% do custo total de produção dos TRAT I, II e III, respectivamente.

O resultado da análise econômica realizada por TRAT, dentro de GG, apresentado nas Tabelas 5 e 6 , revelou que a atividade de confinamento isoladamente tornou-se menos competitiva para os GG que sofreram maior redução no ganho de peso, com o aumento de peso de abate (CA e CN). Esta análise mostrou também que é inviável utilizar animais de baixo peso vivo inicial $(<230 \mathrm{~kg}$ ) e baixo ganho de peso no confinamento (NE), sendo responsável pelo prolongamento do período de confinamento para atingir o peso mínimo de 15 arrobas de carcaça, exigido pela maioria dos programas de incentivo a produção do “Novilho Precoce”, e de 16 arrobas para alcançar preço diferenciado em alguns frigoríficos no Estado de São Paulo.

O problema para comercialização destes animais torna-se mais grave em função da forma de pagamento de animais leves (peso abaixo de $225 \mathrm{~kg}$ de carcaça), que sofrem deságio de $15 \%$, o qual não foi considerado nos cálculos citados nas referidas tabelas. Então, torna-se difícil convencer produtores a produzir carcaças com mais de $3 \mathrm{~mm}$ de gordura externa, antes que a indústria frigorífica estabeleça novos padrões de qualidade e preços diferenciados para estes animais. Atualmente, carcaças acima de $1 \mathrm{~mm}$ de gordura externa são aceitas para exportação e também dentro dos programas estaduais de incentivo à produção do "Novilho Precoce".

Os custos totais de produção por arroba de carcaça vendida, para os animais BN, CA, CN, LN, NE e PN foram 39,48; 41,47; 41,50; 39,26; 44,10 e 39,77 reais, enquanto as rentabilidades mensais, calculadas de manei-

R. Bras. Zootec., v.33, n.3, p.635-645, 2004 
ra diferente daquela apresentada nas tabelas anteriores; isto é, utilizando-se as médias dos preços pagos em setembro, outubro e novembro, pagos pelo mercado em 2001 (IEA, 2002) correspondente ao mês de venda de cada GG, dentro de TRAT, foram 1,9; 0,5; 0,5; 2,2; -2,0 e 2,3\% para os animais BN, CA, CN, LN, $\mathrm{NE}$ e PN, respectivamente. A rentabilidade negativa do confinamento com animais NE pode ser explicada também em face do preço de venda de dois terços dos animais terem sofrido deságio de $15 \%$, por apresentarem carcaças com peso inferior a 15 arrobas.

Quando os cálculos foram realizados com os preços de venda dos animais de $\mathrm{R} \$ 41,86$; $\mathrm{R}$ 44,88 e R\$44,99 por arroba de carcaça nos TRAT I, II e III, respectivamente (média dos preços pagos nos meses de venda de animais de cada tratamento), a rentabilidade mensal foi 0,2; 1,7 e 1,6\%. É interessante observar a redução de rentabilidade do trata- mento II para o III ocorrida no período de 19 dias, mesmo com o aumento de peso de carcaça dos animais de 16,44 para 17,72 arrobas, em função do aumento dos custos da arroba produzida no confinamento de 40,31 para 40,49 reais, sendo arriscado aguardar melhoria de preço no mercado frigorífico para realizar a venda.

Em comparação com os resultados citados na literatura, pode-se observar que, no presente trabalho, os animais cruzados apresentaram melhor resposta de ganho de peso e conversão alimentar, viabilizando o confinamento de animais com 12 meses idade, com a utilização de alimentos de alta qualidade em dietas balanceadas. Os animais NE comerciais, com peso inicial entre 200 e $230 \mathrm{~kg}$ aos 12 meses de idade, mesmo com dietas que propiciam altas taxas de ganho de peso vivo, demonstraram ganho de peso semelhante aos dos outros trabalhos

Tabela 4 - Composição relativa dos custos e rentabilidade da produção do bovino jovem em confinamento, em percentagem do custo do confinamento, e custo da arroba de carcaça produzida e vendida, em reais ( $R$ \$), de acordo com o peso de abate

Table 4 - Relative composition of costs and profitability of production of young bulls in feedlot, in percentage of the feedlot costs, and total costs (R\$), of production of $15 \mathrm{~kg}$ of carcass gain and sold, according to the slaughter weight

\begin{tabular}{|c|c|c|c|}
\hline \multirow[b]{2}{*}{$\begin{array}{l}\text { Itens } \\
\text { Items }\end{array}$} & \multicolumn{3}{|c|}{$\begin{array}{l}\text { Peso de abate, } \mathrm{kg} \\
\text { Slaughter weight, } \mathrm{kg}\end{array}$} \\
\hline & 400 (I) & 440 (II) & 480 (III) \\
\hline $\begin{array}{l}\text { Instalações do confinamento, \% } \\
\text { Feedlot structure, \% }\end{array}$ & 1,3 & 1,0 & 0,9 \\
\hline $\begin{array}{l}\text { Vacinas e medicamentos, \% } \\
\text { Vaccines and drugs, \% }\end{array}$ & 1,1 & 0,9 & 0,8 \\
\hline $\begin{array}{l}\text { Alimentos, \% } \\
\text { Feeds, \% }\end{array}$ & 82,9 & 83,2 & 83,7 \\
\hline $\begin{array}{l}\text { Mão-de-obra para alimentação dos animais, \% } \\
\text { Personel to feed the animals, \% }\end{array}$ & 7,1 & 7,1 & 6,9 \\
\hline $\begin{array}{l}\text { Hora-máquina para alimentação dos animais, \% } \\
\text { Hour of tractor for feeding the animals, \% }\end{array}$ & 4,9 & 4,9 & 4,7 \\
\hline $\begin{array}{l}\text { Assistência veterinária, \% } \\
\text { Veterinary assistance, \% }\end{array}$ & 1,0 & 0,8 & 0,7 \\
\hline $\begin{array}{l}\text { Juros sobre capital de custeio, \% } \\
\text { Interest on invested capital on variable costs, \% }\end{array}$ & 1,7 & 2,1 & 2,4 \\
\hline $\begin{array}{l}\text { Rentabilidade, } \% \\
\text { Net income, } \%\end{array}$ & 3,5 & 2,8 & 3,0 \\
\hline $\begin{array}{l}\text { Rentabilidade mensal, \% } \\
\text { Monthly net income, \% }\end{array}$ & 1,0 & 0,7 & 0,6 \\
\hline $\begin{array}{l}\text { Custo da @ produzida (custeio), R\$ } \\
\text { Cost of production of } 15 \mathrm{~kg} \text { carcass (variable costs), } R \$\end{array}$ & 39,14 & 40,31 & 40,49 \\
\hline $\begin{array}{l}\text { Custo dos alimentos/@ produzida, R\$ } \\
\text { Cost of feeds for production of } 15 \mathrm{~kg} \text { carcass, } R \$\end{array}$ & 33,00 & 34,25 & 34,71 \\
\hline $\begin{array}{l}\text { Custo total por @ vendida, R\$ } \\
\text { Total cost of } 15 \mathrm{~kg} \text { carcass sold, } R \$\end{array}$ & 40,51 & 40,81 & 40,74 \\
\hline
\end{tabular}

R. Bras. Zootec., v.33, n.3, p.635-645, 2004 
Tabela 5 - Custo e rentabilidade de produção do bovino jovem em confinamento, por peso de abate de animais Blonde d'Aquitaine $x$ Nelore, Limousin $x$ Nelore e Piemontês x Nelore

Table 5 - Cost and profitability of production in feedlot of young bull Blonde d'Aquitaine $x$ Nellore, Limousin $x$ Nellore and Piedmontese $x$ Nellore, according to the slaughter weight

\begin{tabular}{lll}
\hline & \multicolumn{2}{c}{$\begin{array}{c}\text { Peso de abate, kg, } \\
\text { Slaughter weight, } k g\end{array}$} \\
\cline { 2 - 2 } Itens & 400 (I) & 440 (II) $\quad 480$ (III) \\
Items & & \\
\hline
\end{tabular}

\section{Blonde d'Aquitaine x Nelore}

Blonde d'Aquitaine x Nellore

Custo da @ produzida (custeio), R\$
Cost of production of $15 \mathrm{~kg}$ carcass (variable costs), $R \$$

Custo da alimentação/@ produzida, R\$

Cost of feeds for production of $15 \mathrm{~kg}$ carcass, $R \$$

Custo total por@vendida, R\$

$37,63 \quad 38,36 \quad 38,58$

Total cost of $15 \mathrm{~kg}$ carcass sold, $R \$$

Rentabilidade, \%

Net income, \%

Rentabilidade mensal, \%

Monthly net income, \%

$30,94 \quad 32,10 \quad 32,38$

\begin{tabular}{|c|c|c|c|}
\hline \multicolumn{4}{|l|}{$\begin{array}{l}\text { Limousin } x \text { Nelore } \\
\text { Limousin } x \text { Nellore }\end{array}$} \\
\hline Custo da@produzida (custeio), R\$ & 37,58 & 36,76 & 40,75 \\
\hline Cost of production of $15 \mathrm{~kg}$ carcass (variable costs), $R \$$ & & & \\
\hline Custo da alimentação/@produzida, R\$ & 30,82 & 30,37 & 33,76 \\
\hline Cost of feeds for production of $15 \mathrm{~kg}$ carcass, $R \$$ & & & \\
\hline Custo total por@vendida, R\$ & 39,71 & 38,79 & 40,17 \\
\hline \multicolumn{4}{|l|}{ Total cost of $15 \mathrm{~kg}$ carcass sold $(R \$)$} \\
\hline Rentabilidade, \% & 5,6 & 8,1 & 4,4 \\
\hline Net income, \% & & & \\
\hline Rentabilidade mensal, \% & 2,2 & 2,6 & 1,0 \\
\hline \multicolumn{4}{|l|}{ Monthly net income, \% } \\
\hline \multicolumn{4}{|l|}{$\begin{array}{l}\text { Piemontês x Nelore } \\
\text { Piedmontese x Nellore }\end{array}$} \\
\hline $\begin{array}{l}\text { Custo da @ produzida (custeio), R\$ } \\
\text { Cost of production of } 15 \text { kg carcass (variable costs), } R \$\end{array}$ & 40,86 & 40,79 & 40,90 \\
\hline $\begin{array}{l}\text { Custo da alimentação/@ produzida, } \mathrm{R} \$ \\
\text { Cost of feeds for production of } 15 \mathrm{~kg} \text { carcass, } R \$\end{array}$ & 32,61 & 32,62 & 33,12 \\
\hline Custo total por@vendida, R\$ & 40,21 & 40,49 & 40,15 \\
\hline Total cost of $15 \mathrm{~kg}$ carcass sold, $R \$$ & & & \\
\hline Rentabilidade, \% & 4,3 & 3,6 & 4,5 \\
\hline Net income, \% & & & \\
\hline Rentabilidade mensal, \% & 1,1 & 0,7 & 0,8 \\
\hline Monthly net income, \% & & & \\
\hline
\end{tabular}

(Galvão et al., 1991; Jorge et al., 1997), tornando o tempo de confinamento prolongado e reduzindo a rentabilidade para este grupo genético. Alternativas de alimentação e manejo, para os animais da raça $\mathrm{NE}$, devem ser estudadas, uma vez que a maior parte dos animais foi abatida com peso vivo menor que o previsto para o tratamento de $440 \mathrm{~kg}$ de peso vivo, por estarem prontos para abate na avaliação visual e também pela baixa taxa de ganho de peso ao final do experimento. 
Tabela 6 - Custo e rentabilidade de produção do bovino jovem em confinamento, por peso de abate de animais Canchim, Canchim x Nelore e Nelore

Table 6 - Cost and profitability of production in feedlot of young bull Canchim, Canchim $x$ Nellore and Nellore, according to the slaughter weight

\begin{tabular}{|c|c|c|c|}
\hline \multirow{3}{*}{$\begin{array}{l}\text { Itens } \\
\text { Items }\end{array}$} & \multicolumn{3}{|c|}{$\begin{array}{l}\text { Peso de abate, kg, } \\
\text { Slaughter weight, kg }\end{array}$} \\
\hline & \multirow[t]{2}{*}{$400(\mathrm{I})$} & \multirow[t]{2}{*}{440 (II) } & \multirow[t]{2}{*}{480 (III) } \\
\hline & & & \\
\hline \multicolumn{4}{|l|}{$\begin{array}{l}\text { Canchim } \\
\text { Canchim }\end{array}$} \\
\hline Custo da @ produzida (custeio), R\$ & 37,75 & 41,95 & 41,99 \\
\hline \multicolumn{4}{|l|}{ Cost of production of $15 \mathrm{~kg}$ carcass (variable costs), $R \$$} \\
\hline Custo da alimentação/@produzida, R\$ & 31,79 & 35,30 & 35,27 \\
\hline \multicolumn{4}{|l|}{ Cost of feeds for production of $15 \mathrm{~kg}$ carcass, $R \$$} \\
\hline Custo total por@vendida, R\$ & 40,63 & 41,69 & 41,64 \\
\hline \multicolumn{4}{|l|}{ Total cost of $15 \mathrm{~kg}$ carcass sold, $R \$$} \\
\hline Rentabilidade, $\%$ & 3,2 & 0,6 & 0,7 \\
\hline \multicolumn{4}{|l|}{ Net income, \% } \\
\hline Rentabilidade mensal, \% & 1,2 & 0,2 & 0,2 \\
\hline \multicolumn{4}{|l|}{ Monthly net income, \% } \\
\hline \multicolumn{4}{|l|}{$\begin{array}{l}\text { Canchim } x \text { Nelore } \\
\text { Canchim } \times \text { Nellore }\end{array}$} \\
\hline Custo da@produzida (custeio), R\$ & 39,27 & 43,96 & 41,58 \\
\hline Cost of production of $15 \mathrm{~kg}$ carcass (variable costs), $R \$$ & & & \\
\hline Custo da alimentação/@produzida, R\$ & 32,81 & 36,82 & 35,09 \\
\hline \multicolumn{4}{|l|}{ Cost of feeds for production of $15 \mathrm{~kg}$ carcass, $R \$$} \\
\hline Custo total por@vendida, R\$ & 40,89 & 42,50 & 40,85 \\
\hline \multicolumn{4}{|l|}{ Total cost of $15 \mathrm{~kg}$ carcass sold, $R \$$} \\
\hline Rentabilidade, $\%$ & 2,5 & $-1,3$ & 2,7 \\
\hline \multicolumn{4}{|l|}{ Net income, \% } \\
\hline Rentabilidade mensal, \% & 0,9 & $-0,3$ & 0,6 \\
\hline \multicolumn{4}{|l|}{ Monthly net income, \% } \\
\hline $\begin{array}{l}\text { Nelore } \\
\text { Nellore }\end{array}$ & & & \\
\hline Custo da@produzida (custeio), R\$ & 47,75 & 49,04 & 49,11 \\
\hline \multicolumn{4}{|l|}{ Cost of production of $15 \mathrm{~kg}$ carcass (variable costs), $R \$$} \\
\hline Custo da alimentação/@produzida, R\$ & 37,39 & 38,32 & 38,52 \\
\hline \multicolumn{4}{|l|}{ Cost of feeds for production of $15 \mathrm{~kg}$ carcass, $R \$$} \\
\hline Custo total por@vendida, R\$ & 44,34 & 44,93 & 44,86 \\
\hline \multicolumn{4}{|l|}{ Total cost of $15 \mathrm{~kg}$ carcass sold, $R \$$} \\
\hline Rentabilidade, $\%$ & $-5,5$ & $-6,7$ & $-6,5$ \\
\hline Net income, \% & & & \\
\hline Rentabilidade mensal, \% & $-1,1$ & $-1,2$ & $-1,1$ \\
\hline Monthly net income, \% & & & \\
\hline
\end{tabular}

\section{Conclusões}

O aumento do peso de abate de bovinos nãocastrados causa redução no ganho diário de peso, no consumo de matéria seca, expresso em percentagem do peso vivo, na conversão alimentar e na rentabilidade do confinamento.

Os dados sugerem que o confinamento é uma atividade que requer uma gestão eficiente para aliar o melhor peso de abate ao maior retorno econômico.

\section{Agradecimento}

Aos produtores rurais Amilcar Farid Yamin, Antônio Villares da Silva Novaes, José Ferreira Fernandes e Rubens de Assumpção, pela parceria na execução dos trabalhos do confinamento.

\section{Literatura Citada}

BAILEY, C.M.; LIBORIUSSEN, T.; ANDERSEN, H.R. et al. Producing beef from intact male progeny of Holstein sires: feed efficiency and compositional characters. Journal Animal

\section{R. Bras. Zootec., v.33, n.3, p.635-645, 2004}


Science, v.61, n.1, p.27-35, 1985.

BOLETIM DO LEITE. Custo de produção de silagem de milho. Boletim do Leite, v.8, n.91, p.3, 2001.

BROADBENT, P.J. Growth and carcass characteristics of weaned single-suckled calves slaughtered on achieving slaughter condition or 8 or 16 weeks later. Animal Production, v.23, n.2, p.155-163, 1976.

CRUZ, G.M. Produção de carne bovina utilizando confinamento. In: SIMPÓSIO SOBRE MANEJO E NUTRIÇÃO DE GADO DE CORTE, 1., 2000, Goiânia. Anais... Goiânia: Colégio Brasileiro de Nutrição Animal, 2000. p.91-106.

EUCLIDES FILHO, K.; EUCLIDES, V.P.B.; FIGUEIREDO, G.R. et al. Efeito da suplementação com concentrado sobre idade de abate e características de carcaça de bovinos Nelore. Revista Brasileira de Zootecnia, v.26, n.6, p.1096-1102, 1997.

GALVÃO, J.G.; FONTES, C.A.A.; PIRES, C.C. et al. Ganho de peso, consumo e conversão alimentar em bovinos nãocastrados, de três grupos raciais, abatidos em diferentes estágios de maturidade (Estudo I). Revista da Sociedade Brasileira de Zootecnia, v.20, n.5, p.494-501, 1991.

GOHERING, H.K.; Van SOEST, P.J. Forage fiber analyses. Apparatus, reagents, procedures and some applications: Agricultural Research Service/United States Department of Agriculture, 1970. 45p (Agricultural Handbook, 379).

HUFFMAN, R.D.; WILLIAMS, S.E.; HARGROVE, D.D. et al. Effects of percentage Brahman and Angus breeding, ageseason of feeding and slaughter end point on feedlot performance and carcass characteristics. Journal of Animal Science, v.68, n.8, p.2243-2252, 1990.

INSTITUTO DE ECONOMIA AGRÍCOLA. Banco de dados: preços médios pagos pela agricultura. Disponível em: http://www.iea.sp.gov.br. Acesso em: 10 de junho, 2002.

JORGE, A.M.; FONTES, C.A.A.; FREITAS, J.A. et al. Ganho de peso e de carcaça, consumo e conversão alimentar de bovinos e bubalinos, abatidos em dois estágios de maturidade. Revista Brasileira de Zootecnia, v.26, n.4, p.806-812, 1997.

LEVY, D.; HOLZER, Z.; FOLMAN, Y. Effect of concentrate: roughage ratio on the production of beef from israeli-friesian bulls slaughtered at different live weights. Animal Production, v.20, n.3, p.199-205, 1975.
MATTOS, J.C.A. Programa de produção de carne qualificada de bovídeos do Estado de São Paulo (Novilho Precoce). In: ENCONTRO NACIONAL SOBRE NOVILHO PRECOCE, 1., 1995, Campinas. Anais... Campinas: CATI, 1995. p.13-21.

McCULLOUGH, M.E. Silage - some general considerations. In: McCULLOUGH, M.E. (Ed.) Fermentation of silage - a review. Des Moines: National Feed Ingredients Association, 1978, p.1-26.

NATIONAL RESEARCH COUNCIL - NRC. Nutrient requirements of beef cattle. 7.ed. Washington, D.C. National Academy of Science, 1996. 242p.

PATTERSON, D.C.; MOORE, C.A.; STEEN, R.W.J. The effects of plane of nutrition and slaughter weight on the performance and carcass composition of continental beef bulls given high forage diets. Animal Production, v.58, n.1, p.41-47, 1994.

PRESTON, T.R.; WILLIS, M.B. Intensive beef production. 2.ed. Oxford: Pergamon Press, 1974. 567p.

STATISTICAL ANALYSES SYSTEM - SAS. User's guide: Statistics. Cary: 1999. Site 0030966035.

SILVA, D.J. Análise de alimentos: métodos químicos e biológicos. Viçosa, MG: Universidade Federal de Viçosa, 1990. 165p.

URICK, J.J.; MacNEIL, M.D.; REYNOLDS, W.L. Biological type on postweaning growth, feed efficiency and carcass characteristics of steers. Journal of Animal Science, v.69, n.2, p.490-497, 1991.

VILELA, D. Aditivos para silagens de plantas de clima tropical. In: REUNIÃO ANUAL DA SOCIEDADE BRASILEIRA DE ZOOTECNIA, 35.; SIMPÓSIO ADITIVOS NA PRODUÇÃO DE RUMINANTES, 1., 1998, Botucatu. Anais... Botucatu: Sociedade Brasileira de Zootecnia, 1998. p.73-108.

Recebido em: 05/09/02

Aceito em: 10/09/03 